This item was submitted to Loughborough's Institutional Repository (https://dspace.lboro.ac.uk/) by the author and is made available under the following Creative Commons Licence conditions.

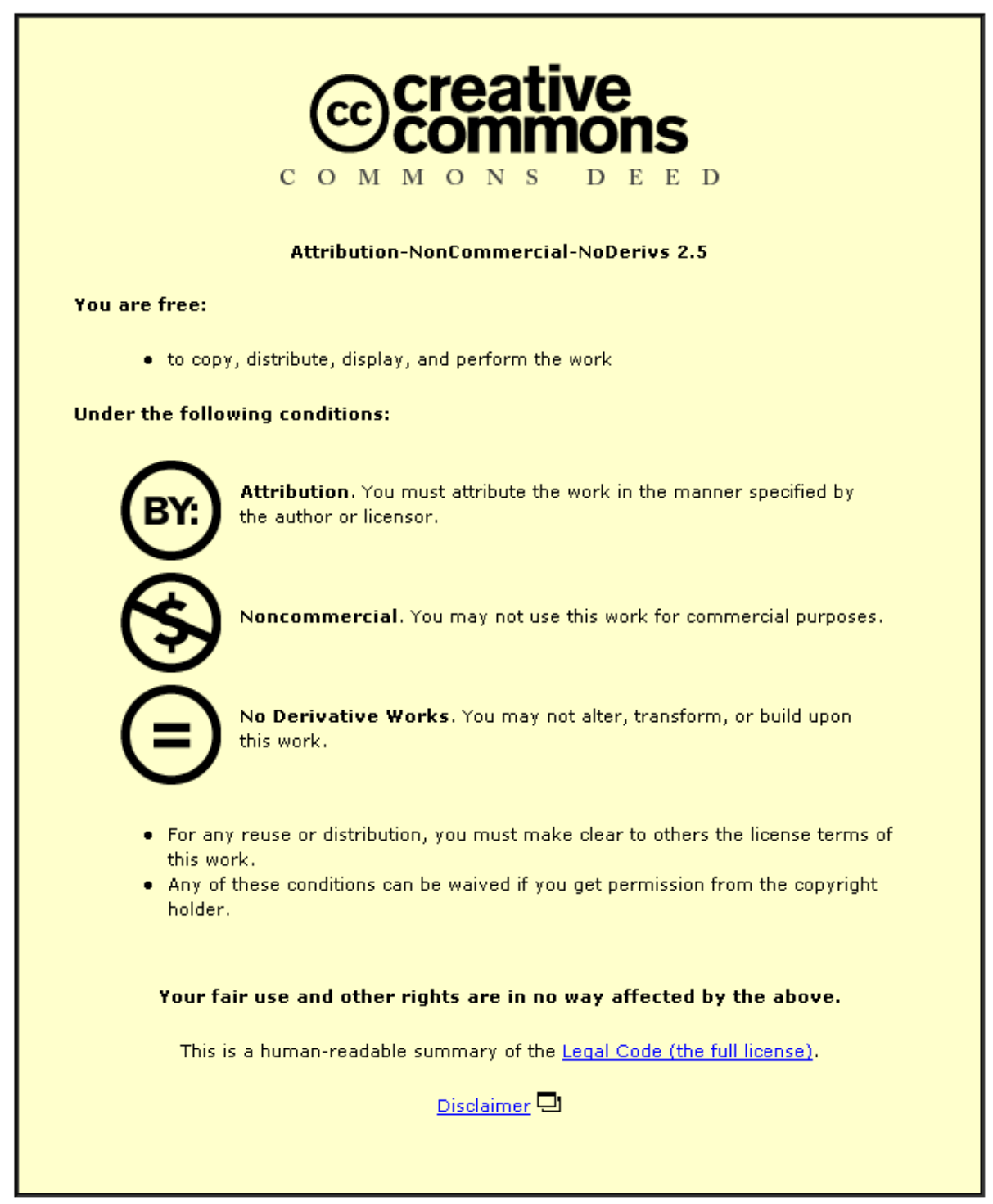

For the full text of this licence, please go to: http://creativecommons.org/licenses/by-nc-nd/2.5/ 


\title{
The Consideration of Organizational Issues during the Systems Development Process: An Empirical Analysis
}

\author{
N. F. DOHERTY, M. KING \\ (Loughborough University Business School, \\ Loughborough, LE11 3TU)
}

Short Title: Organizational Issues in Systems Development

Mailing Address:

Professor Malcolm King,

Loughborough University Business School,

Loughborough University of Technology,

Loughborough, LE11 3TU,

United Kingdom.

Telephone: 01509223119

Email: M.King@lboro.AC.UK

Fax:

01509269332

Email: N.F.Doherty@ Iboro.AC.UK 


\section{The Consideration of Organizational Issues during the Systems Development Process: An Empirical Analysis}

Key Words: Systems development; Systems failure; Organizational issues; United Kingdom.

Short Title: Organizational Issues in Systems Development

Abstract: The lack of consideration of organizational issues in systems development can lead to project failure. A review of the literature and a pre-test survey suggested classifying organizational issues into five categories and examining how these are considered by IT managers. A postal survey with responses from 64 senior IT specialists over a cross section of industry and commerce showed that there is a general awareness of the importance of organizational issues but there was little consensus on how they should be addressed in the development process. These IT managers were consistent in spending most effort on the issues perceived as most important from the list of fourteen issues provided, but there was considerable variation in which specific issues they rated most important. In general those organizational issues with a "technical" aspect were given more prominence than those which are less tangible, but which may be more critical to a system's success. 


\section{Introduction}

A large number of information systems development projects can be classified as either complete or partial failures because they are either excessively over budget, months or years behind schedule, of poor quality, or simply because they fail adequately to satisfy users' requirements. Lyytinen and Hirschiem (1987), for example suggest that more than $50 \%$ of all systems development projects end in failure. There is also a growing realisation that it is often organizational issues that play a substantial, if not the primary, role in systems failure. (Long 1987; Kearney 1990; Buchanan 1991; Ewusi-Mensah \& Przasnyski 1994). Concern with the relationship between information systems failure and organizational issues, is not, however, a new phenomena. Indeed, Henry Lucas (1975) wrote a classic book over twenty years ago, in which he suggested that: 'the primary cause for systems' failure has been organizational behaviour problems'.

There is, however, much recent evidence to suggest that organizational issues are now more important, and will become even more critical, to the successful development and implementation of information systems than they were when Henry Lucas wrote his book. In the past, information systems were primarily developed to support the administrative functions within a business, by simply automating existing processes, without fundamentally changing the way in which an organization operated. The current trend is for information systems which are highly integrated, have the ability to disseminate information throughout an organization, and often have become an integral part of the actual product or service provided. Such trends, which have been summarised in Table 1, mean that new information systems may bring about changes to an organization's structure, working practices, and culture, all of which in turn alter the way in which power and resources are distributed throughout the organization (Stowell \& Weat 1994).

\section{Table 1 about here}

Given the recognised importance of organizational issues, it is somewhat surprising that much recent research suggests that systems development is still 'technology led' (Clegg et al, 1994), that organizational issues are not properly addressed during the systems development process (Clegg et al, 1996), and that much of the responsibility for this rests with IT professionals (Hornby et al 1992). There is, therefore, a pressing need for a broad programme of research to consider why organizational issues are given a low priority, and how their treatment can best be accommodated. An important introductory part of this research is to ascertain the current views of senior IT professionals with regard to the treatment and importance of organizational issues, both at a macro and a micro level. The overall aim, therefore, of this paper is to investigate the attitudes of senior IT managers to the importance, and treatment, of organizational issues in general, and then to build a picture of how they address specific organizational issues, and which of these issues they consider to be of the most importance. To achieve this it is necessary to build a comprehensive list of specific issues, arranged in a clear framework. 


\section{A Review of the Treatment of Organizational Issues in the Systems Development Process}

At the out-set it is necessary to clarify what is meant by the term organizational issues, and then to review how such issues are typically treated in the systems development process.

\subsection{What are Organizational Issues?}

Although there have been few attempts to explicitly define the term organizational issue, they have been defined by providing examples of 'non-technical' aspects of systems development, which might have an impact on the ultimate success or failure of a project (Eason 1988; Clegg et al 1989). An analysis of these examples suggests that it is possible to categorise them into three groups, as follows:

\subsubsection{Organizational Alignment}

The term 'organizational validity' originally coined by Schultz and Slevin (1975), is typically used to describe 'the degree of fit or match between a system and its organizational context' (Markus \& Robey 1983). For the purposes of this analysis, however, the term 'organizational alignment' is preferred, as this better expresses the concept of fitting or matching. It is now recognised that this closeness of fit between a system and its host organization is critical to its ultimate success (Walton 1989; Pliskin et al 1993).

There are three main proactive ways in which a new system can be successfully aligned with its organizational environment. Firstly, systems development projects can be used as an explicit mechanism for re-designing an organization (Buchanan 1991). Alternatively, the existing organizational context can be viewed as fixed, and the new system can be designed, so that it matches its host organizational environment (Pliskin et al 1993). Finally, it can be recognised that there must be 'organizational adaptation to complement technological change' (Willcocks \& Griffiths 1994), and, therefore, the level of organizational adaptation necessary must be evaluated in advance to ensure that it is desirable. The alternative to proactive alignment, is reactive alignment, whereby a system is developed and implemented, and the organizational implications are only considered, as and when problems arise. Unfortunately there is much evidence to suggest that most organizations, by default, opt for reactive alignment (Hornby et al 1992).

Several authors identify specific issues which should be considered when aligning a system. For example, the proposed system needs to be correctly aligned with the organizational structure (Markus \& Robey 1983; Harrington 1991; Raymond et al 1995), the power distribution (Markus \& Robey 1983; Sauer 1993; Thach \& Woodman 1994), and the organizational culture (Walton 1989; Romm et al 1991; Pliskin et al 1993). It is clear from this literature that whilst a system can be designed to make a significant positive contribution to an organization's performance, if it is not carefully aligned with the structure, culture and power distribution of the host organization, then it may fail to deliver its full potential, or may even fail completely. 


\subsubsection{Organizational contribution}

This group of issues includes those which focus on ensuring that a proposed system will make a positive contribution to the performance of some part the organization. This might be in the form of contributing to the financial, marketing, or operational performance of the organization. Probably the most widely accepted way of doing this is through pro-active user involvement (Wong \& Tate 1994). Indeed, Hornby et al (1992), have reported that many systems analysts assume that by involving end-users they will have implicitly addressed all the 'salient organizational and human issues'. It is possible, however, to build a system which is based upon some user wants, but which fails to make a positive contribution to the overall performance of the organization.

There are other issues which need to be addressed to ensure that a system makes a positive contribution to the organization as a whole. The creation of an information systems strategy to guide the selection and development of all information systems, is a key factor (Earl 1989; Coombs 1992). The completion of a thorough cost-benefit analysis (Flatten et al 1991), should also help to ensure that the development effort is focused upon overall contribution to the organization's objectives. Furthermore, as information systems operate in a highly dynamic environment, it is also important that the future needs of the organization (Crowe et al, 1996) are explicitly considered. It can be seen that this group covers issues which are more concerned with the purpose of the system, and how that might contribute to organizational objectives, rather than being concerned with the environment in which the system might operate.

\subsubsection{Human Issues}

Human aspects of systems development are those issues which have a discernible impact on the working practices and environment of individual employees, who directly interact with the system. For example, issues such as user training, user motivation, human-computer interface design, and ergonomic and health \& safety issues, can all be considered human in orientation. Furthermore, the re-design of jobs (Eason 1988), in parallel with the design of a new system is also of great importance to its ultimate success. Although it is possible to differentiate between such human issues, which relate to those individuals who have primary operational interaction with the system, and wider organizational issues which have an impact on the organization as a whole, some researchers (e.g.: Horny et al 1992; Lucas 1975) choose not to differentiate between them because they are all 'non-technical' in orientation.

It should be noted that, most of the issues listed under 'organizational contribution' and 'human issues' have both organizational and technical dimensions. For example, user's participation in systems development is encouraged, at least in part, so that an appropriate technical specification can be derived; an information systems strategy is created to ensure that the technical infrastructure will support systems which provide a measurable organizational contribution; and user interface design is concerned with both technical and human aspects of human-computer interaction. 


\title{
2.2 The Treatment of Organizational Issues in Systems Development Projects
}

The vast majority of literature published on systems development focuses on either development methodologies, or technical aspects of design. The breadth and depth of this body of literature is vast, and by comparison the literature on the treatment of organizational issues within systems development is trivial. This is probably because the systems development process is still primarily a technology driven process. As Hornby et al (1992) note:

\begin{abstract}
'Systems analysts do not claim to have knowledge of organizational issues in IT systems, and there is no evidence that they are encouraged or rewarded for considering such issues. In fact it could be said that the reward and control systems within which analysts work actively encourage them not to consider them. They are rewarded in the main, for delivering technically sound systems on time and to budget'.
\end{abstract}

The technical orientation of systems development methodologies has typically resulted in the approach of implementing a system, and then trying to cope with its organizational implications.

Eason(1988) notes that the structured design methods, which are the most common means of developing complex, centralised systems, are typically devoted to the design of technical systems and give very little recognition, or support, to the range of organizational changes that also have to be designed. The work of Hornby et al (1992) supports this finding, in that common development methodologies, such as SSADM, Yourdon and SSAD, are still very technically orientated, with their treatment of organizational issues going very little beyond urging developers to involve the users. Methodologies, which are more organizationally focused, such as ETHICS (Mumford 1986), are, by comparison, rarely used by IT professionals. There is also the recognition that the aspects of development, which have been categorised as 'organizational alignment' issues in this paper, are, in particular, largely ignored by systems analysts, as they are considered to be the responsibility of the user management representatives.

Having reviewed some of the most important contributions to the literature in this area, it is possible to establish how the research presented in this paper, builds upon what has gone before. Much of the cited literature examines the importance, and treatment, of a single organizational issue, for example organizational power (Bloomfield \& Coombs, 1992); organizational culture (Walton, 1989) or information systems strategy (Earl, 1989). This paper, however, seeks to review and compare the relative importance, and treatment of a wide range of different specific organizational issues. Hornby et al (1992), have already explored how organizational issues are addressed in a range of systems development methodologies. However, it may very well be that organizational issues are treated outside the confines of such methods. This research, therefore, builds on the work of Hornby et al (1992) in that it investigates how organizational issues are treated in practice, irrespective of the development methodology used, and it also compares their relative importance to the ultimate success and failure of systems development projects. 


\section{Research Method}

To canvass the views and perceptions of a wide range of IT managers a draft questionnaire was developed, based primarily on issues that had been highlighted as being of interest from the literature review. This draft questionnaire was then thoroughly validated by pre-testing it with IT managers from five local companies who were willing both to complete it, and then discuss in detail its content, and ease of use. These managers all agreed that the research was of great interest and highly important in that organizational issues were generally under-valued. They were largely happy with the content of the questionnaire, although a number of interesting enhancements were suggested and ultimately accepted. Their major concern was, however, how willing IT professionals would be to answer questions on a subject were their performance has been generally poor to date, and there was, consequently, concern about likely response rates.

Because of these concerns it was decided to target only those organizations where there were either existing research, or teaching contacts, and therefore the chances of ensuring a positive response were greater. The questionnaire was ultimately distributed to 88 named IT professionals in a wide variety of UK-based organizations. It was recognised that only those individuals who had a high degree of managerial responsibility for systems development projects would be able to comment knowledgeably about the importance of organizational issues, and the extent to which they are routinely addressed. The questionnaire was, therefore only targeted at IT Directors, IT Managers, or Senior Systems Analysts / Consultants who had had direct project management responsibility. A total of 64 valid responses were received from the original sample of 88 ; a highly encouraging response rate of $73 \%$. Five of the respondents were interviewed in addition to completing the questionnaire, which allowed the researchers to probe the thinking behind some the answers to specific questions. Although this survey guaranteed confidentiality, respondents were offered the opportunity to receive a copy of the results if they were interested. Indeed over $80 \%$ of the respondents requested a copy of the results, which indicates, at least in part, the high degree of interest in this topic.

The Questionnaire was divided into four sections each of which is briefly discussed below:

\subsection{Background Information}

Respondents were asked to categorise their organization in terms of size and the sector in which it primarily operates, using standard classifications. Furthermore, the respondents were asked to indicate the level to which their organizations designed and coded their systems in-house, as opposed to using packages or external consultants.

\subsection{Organizational Issues in the Systems Development Process}

This section of the survey focused on two critical issues. Firstly, the relative importance of organizational issues, in comparison to technical issues, when determining the ultimate success or failure of a systems development project, and secondly the method by which organizational issues are addressed during such a project. 
Respondents were offered a number of alternatives when answering these two questions. The precise wording of the alternatives offered is almost identical to the phrases shown on the lefthand side in Figures 1 and 2. Clearly in answering such questions it is important that the respondents have a shared understanding of the difference between organizational and technical issues. To focus respondents minds on the meaning of the term organizational issue, prior to answering these questions, several examples of such issues were presented in the covering letter. Furthermore, the next question listed 14 such issues in a full page spread which was clearly visible when answering the earlier questions. In pre-testing all the respondents seemed confident that there was a common understanding of what was meant by these issues. Similarly, there was some concern over the words 'explicit' and 'implicit', with respect to the treatment of organizational issues, in the second of these questions. These were not defined in the questionnaire but the pre-test group had no difficulty in associating 'explicit' with situations where there was a formal procedure for treating issues, and 'implicit' with instances where this was not the case.

\subsection{Organizational Issues Addressed}

The primary aims of the third section of the survey, were twofold:

(1) To assess the relative importance of different types of organizational issues, in order to identify which specific issues, or groups of issues, are perceived by IT managers to be of most importance to the ultimate success or failure of a systems development project, in the perception of IT managers.

(2) To explore how regularly specific organizational issues are addressed and to identify which are the most commonly considered.

To achieve these two objectives a representative, but not definitive, list of organizational issues was included in the survey. In order to encourage responses, the main body of the questionnaire was deliberately limited to two sides of $A 4$, so that its completion would not be too time consuming, or taxing. Consequently, the list of organizational issues was restricted (14 issues were ultimately selected), and some issues had to be omitted. In determining which issues to include or exclude, care was taken to ensure a representative sample of issues was included from each of the three categories defined in Section 2. Furthermore, it was concluded that it would be of more interest to include those issues which have received less detailed analysis in the past. Consequently, issues such as user participation (Wong \& Tate 1994), and humancomputer interface design (Preece 1994) which have, at least in part, a strong organizational orientation, were deliberately excluded from this section, because their importance, and treatment, has been discussed in great detail elsewhere.

For each of the fourteen organizational issues in the list, the respondents were asked to consider the frequency to which each is routinely addressed during the systems development project (ranging from never to always), and also to consider the relative importance of each specific organizational issue (ranging from 1 to 14). The list of fourteen organizational issues ultimately employed could be sub-divided into the following five major broad groupings (three already highlighted, plus two identified during the pre-test study): 
(1) Organizational Alignment: This section focused upon the extent to which a proposed system and its host organization were matched. Respondents were, therefore, asked whether the relationship between the proposed system and the organization's culture, structure, and the distribution of power were considered.

(2) Organizational Contribution: These questions related to the extent to which a proposed system will make a significant positive contribution to the organization. Consequently, respondents were asked about the use of cost-benefit analysis and information systems strategies, the prioritization of work to reflect organizational requirements, and to what extent the future needs of the organization were considered.

(3) Human Issues: This section focused upon whether human issues are adequately addressed in the systems development process. The questions, therefore related to issues such as training, job re-design and the consideration of health and safety I ergonomic factors. IT managers who participated in the pre-test study also stressed the importance of wider management and user education, and the need to take account of user motivation.

(4) Transitional Issues: These questions related to whether practical transitional issues were addressed, focusing upon issues such as the timing of an implementation, and the perceived level of organizational disruption. It is interesting to note, that whilst the issues contained in the previous three sections had been referenced in the published literature, the issues in this section were suggested by the IT managers who participated in the pre-test survey. It can be argued that these transitional issues may have a significant impact on the long term success or failure of the system.

(5) Systems Integration: A further issue raised during the pre-test testing of the survey, was the level of systems integration which has a direct impact on the dissemination, and utilisation, of information, within an organization. Although the importance of systems integration, and the implementation of enterprise-wide infrastructures has been highlighted (Price Waterhouse, 1996), it has not previously been cited as an example of an organizational issue. The Respondents were, therefore, asked to consider the importance of reviewing how the proposed system would interface to existing systems, and the extent to which this issue was addressed during systems development projects.

The groupings are not intended to represent discrete, and clearly bounded groups, as there is often some degree of overlap between the groups. Rather the groupings are intended to summarise the most important characteristics of organizational issues. It should be noted that these five broad groupings were not highlighted on the questionnaire, as it was thought that this might unduly influence the results.

A third, but less explicit, aim of this section of the questionnaire was to learn more about the issues which could be considered to be organizational in orientation, by identifying issues which have not been not, as yet, been highlighted in the literature. It was envisaged that this could best be achieved by inviting respondents to add other organizational issues, that they believed to be of importance, to the original list of fourteen issues. 


\subsection{Additional Comments}

In addition to the three major sections, discussed above, respondents were invited to use a comments section, to make additional observations.

\section{Research Results}

The results are discussed using the four major sections that were used to structure the questionnaire.

\subsection{Background Information}

The questionnaire was targeted primarily at large organizations, as it was believed that these would have greater experience of developing and implementing a wide range of systems, and also it was envisaged that they would be more likely to have formalised procedures for dealing with organizational issues. Of the 64 responses received 39 (61\%) emanated from large organizations (>2000 employees), whilst the remaining 25 responses were fairly evenly distributed from the other three categories. The majority of the respondents came from the service sector (53\%), whilst a further 38\% came from manufacturing, with the remaining $9 \%$ coming from the retailing sector. Furthermore, by analysing the affiliations of respondents, it was also possible to establish that the majority of the responses originated from high profile and highly influential organizations, whose views and experiences should be of great interest to others.

When it came to the proportion of information systems designed and coded in-house, as opposed to being substantially based upon off-the-shelf, applications packages, or developed by external consultants, the respondents were asked to choose between one of six percentages. It is interesting to note that there were only a small number of outliers, that is organizations who develop either all, or none, of their own systems in house. The remainder of the sample was fairly evenly split between those organizations which develop either $20 \%, 40 \%, 60 \%$, or $80 \%$, inhouse.

\subsection{Organizational Issues in the Systems Development Process}

The question relating to the relative importance of organizational and technical issues when it comes to determining the ultimate success or failure of an information systems development project, probably throws up the most interesting result of this research. Whilst most previous research has indicated that the focus of most systems development methodologies is technical issues at the expense of organizational issues, a significant majority of the respondents to this research felt that organizational issues are more important than technical issues. The majority of the respondents (56\%) perceived that organizational issues were either 'the most important issue', or 'more important than technical issues', whilst a further $38 \%$ felt that organizational issues were 'of equal important to technical issues'. Therefore, only $6 \%$ of the respondents considered technical issues to be of more importance to organizational issues. Figure 1 provides a full break down of the responses to this question. 
There are, perhaps, two interpretations of these findings. Respondents may either honestly believe that organizational issues are more important than technical issues, or they may state that organizational issues are more important because they feel that this is the right answer. Either way, these results demonstrate that IT professionals are now fully aware of the growing importance organizational issues. At least, this indicates that failures to consider organizational issues are not because IT professionals are unaware of their significance.

\section{Figure 1 about here}

The approaches organizations take to addressing organizational issues vary greatly as can be seen from Figure 2. A surprisingly high proportion of the responding organizations, treat organizational issues explicitly (42\%), either during the feasibility study, requirements analysis or through an independent organizational impact analysis. Those organizations which treat organizational issues implicitly is still, however, the largest single category with $49 \%$ of the responses. There must be some concern that the 'implicit' treatment of organizational issues, may fail to adequately address many important issues. Finally, a worrying $9 \%$ of the responding organizations rarely consider such issues at all.

\section{Figure 2 about here}

\subsection{Organizational Issues Addressed}

The analysis of the data, relating to the relative importance, and frequency of consideration, for specific organizational issues, produced some highly interesting results. For each of the fourteen issues, an average 'frequency of use' rating has been calculated, by using a scale of 1 to 5 (where $5=$ always and $1=$ never). The fourteen issues were then arranged in descending order of this average, as shown in Table 2, which also records the minimum and maximum values for each issue, and the number of responses in the categories $1 \& 2$, and $4 \& 5$. As can be seen from Table 2, there is a clear ranking of these issues which indicates a high degree of variation when it comes to the proportion of systems development projects in which each issue is addressed. Some issues, for example 'the need to consider systems interfaces' are nearly always addressed, whilst others, such as evaluating of the impact of a system on 'the distribution of power' are rarely explicitly considered. The explanation for this might very well be that IT professionals concentrate on those issues that they feel best equipped to deal with, and which are least politically sensitive.

\section{Table 2 about here}

Similarly, an average 'importance of issue' rating was calculated using a scale of 1 to 14 (where $1=$ most important, $14=$ least important). Again the fourteen issues were arranged in descending order of this average, as shown in Table 3, which also shows the minimum and maximum values for each issue, and the number of responses in the categories 1-5, and 11-14. From Table 3, it can be seen that there is a clear ranking at either end of the spectrum, but some bunching of those issues in the intermediate ranks. The need to undertake a cost-benefit 
analysis is widely regarded as being of importance, whilst the need to evaluate a systems impact on the distribution of power is perceived to be of far less importance. It is, however, interesting to note from the results that whilst there are some discernible trends in the data, there is also a high degree of variability in peoples views and experiences. For example, the minimum and maximum values for the relative importance of specific issues, covers the full range of values in all but a few instances.

\section{Table 3 about here}

The results relating to organizational issues addressed, and the frequency with which each is addressed, have also been presented together in Figure 3. Each of the 'importance of issue' ratings has then been converted using an appropriate transformation function, so that the value is translated from a 1 to 14 scale to a scale of 1 to 5 . This allows the average values for both 'frequency of use' and 'importance of issue' to be shown on the same graph and their values to be directly compared, so that the degree of correlation between the two results can be visually assessed.

\section{Figure 3 about here}

It is possible to make some general comments with regard to the results presented in Figure 3. Firstly, there is a high degree of correlation between the relative level of importance of an issue, and the frequency with which it is considered, which indicates that effort is being expended in those area which are considered to be of most importance. Furthermore, it is interesting to note that whilst some issues, such as performing a cost-benefit analysis, are considered to be important and addressed in the vast majority of projects, others, in particular the distribution of power, are considered relatively unimportant, and are consequently rarely addressed.

A more detailed review of these findings, presented in Figure 3 , can best be achieved by returning to the five categories utilised in the previous section of this paper:

(1) Organizational Alignment: The data clearly suggests that the 'organizational alignment' issues are generally perceived to be of least importance, and consequently infrequently addressed. This finding is of particular importance, because it is these issues, in particular, which are increasingly being cited (Markus \& Robey 1983; Pliskin et al 1993) as an important contributory factor to systems development failures.

(2) Organizational Contribution: With regard to the relative importance of organizational issues, it is interesting to note that those classified as 'organizational contribution' issues, are clearly considered to be of the greatest importance to the successful development of information systems. Consequently, it is also these issues which are most frequently addressed in systems development projects. The fact that cost benefit analysis attains the highest overall importance rating is, however, disturbing as it has often been criticised for being an imprecise and often inadequate technique (Willcocks 1990; Lincoln \& Shorrock 1990). 
(3) Human Issues: The human issues are generally to be found occupying the middle ground. Training / education, in particular, is considered to be of some importance, whilst health \& safety / ergonomic considerations are generally considered to be of far less importance.

(4) Transitional Issues: The transitional issues are also generally to be found occupying the middle ground. Their relative importance is of much interest, in that they are a set of issues which have, hitherto, received little attention. Both the timing of implementation, and to a lesser extent the level organizational disruption, are considered to be of some importance.

(5) Systems Integration: The data with regard to the need for considering interfaces is of intriguing because of its apparent inconsistency. Whilst it is perceived to be of relatively little importance, systems interfaces are almost always considered. This may very well be because most developers, only consider them at a technical level (i.e. is there any existing data that can be easily captured), rather than considering their broader organizational implications.

Many respondents accepted the opportunity to add organizational issues which they felt to be of importance, but which had not been explicitly cited in the questionnaire. Of the many additional issues suggested the following were the most common: assessing the system's impact on external stakeholders, especially customers; the phasing out of existing systems; the role and composition of support groups; the need to conduct risk assessment; the need to take account of the strategic direction of the host organization; and the need to address organizational issues during the post-implementation review. It was interesting to note that only one respondent considered that user participation is an organizational issue which should have, therefore, been included in the original list.

\subsection{Additional Comments}

Many interesting findings came from the additional comments offered by the respondents. There were, for example, numerous comments noting that systems development is still primarily a technical exercise, but stressing the importance of organizational issues to a project's ultimate success or failure. The following is a summary of some of the other more common and interesting comments:

Organizational issues difficult to address: Despite their acknowledged importance organizational issues are generally difficult to address, because they are less tangible than technical issues. The 'organizational alignment' issues are, in particular, often difficult to explicitly address, because they are both highly unpredictable and politically sensitive. Furthermore, because of the tight time and cost constraints within which systems are developed, it is likely that organizational issues will be ignored in the interests of developing a technically sound system, on time and within budget.

Dynamic environment: Information systems are operating in an increasingly dynamic environment. It is, therefore, essential that the organizational issues with regard to future needs are thoroughly examined, and translated into a technical specification, which is sufficiently flexible. One respondent, however, commented on the difficulty of getting users to focus on the broader issues rather than just their immediate needs. 
The importance of organizational buy-in: Organizational issues are far better addressed in circumstances where the organization takes strong, and highly visible, ownership of the project, and guides the system development activity.

Organizational issues and project size: The importance of organizational issues is directly proportional to the size of the project, and they are, therefore, often ignored on small PC-based applications. There is, however, a danger in this, as on respondent pointed out, in that numerous ' $P C$ by $P C$ implementations' can bring about drastic organizational changes without their impact being properly assessed.

Organizational issues and packages: When purchasing 'off the shelf' packages, organizational issues are often more important, and more readily considered, in that the organization often has to adapt to the package, rather than the system being tailored to the organization.

Organizational issues and Business Process Reengineering: Where IT developments are being initiated in conjunction with a re-engineering programme, organizational issues are often more thoroughly addressed, in that it is organizational change that is driving systems projects, and not the reverse.

\section{Conclusions}

This paper presents an initial, quantitative study of an important area of research, which has so far been rather neglected, in terms of surveys of practitioners. The results of this survey should be of general interest, in that they represent the views of a wide variety of IT professionals, with management responsibilities, who are generally employed by highly influential organizations.

The finding that the vast majority of these respondents considered organizational issues to be of equal, if not greater importance to technical issues, supports the work of others who have approached this area from different perspectives. The significance of this finding, is however, heightened, in that it is IT professionals who are now recognising the importance of organizational issues; a group who have traditionally focused upon technical factors, at the expense of organizational ones. The fact that cost-benefit analysis, an often maligned technique, is rated as the most important organizational issue, perhaps provides an insight into this apparent anomaly. This might be because whilst at a global level, IT professionals recognise the importance of organizational issues, when it comes to addressing specific organizational issues, they haven't got the necessary tools and techniques, and consequently they tend to concentrate on the more tangible organizational issues.

The high degree of correlation between respective levels of importance, and frequency of consideration, for the fourteen specific organizational issues, is also generally reassuring. It probably suggests that the level of importance determines the frequency of treatment, although there is also the possibility that specific issues are perceived to be important because they are regularly considered. Furthermore, it is interesting to note that the 'organizational alignment' issues, which are the most commonly cited, and the most obviously organizational in orientation, should be generally rated as the least important, and the least considered, by practitioners. This is very probably because they are the least tangible of all of the organizational issues, they will 
generally be outside the realm of expertise of the majority of IT professionals, and they can be extremely politically sensitive. Since many writers now argue that these are the most critical issues relating to a system's success, it is very important for future research to establish if these are the reasons, or if there is some other explanation.

Whilst the identification of a definitive list of organizational issues, was not one of the primary aims of this research, the following points emerged:

- IT managers are widening their views to include organizational issues, such as assessing the needs of external stakeholders, and the need to prioritize the systems development activity, so that effort is concentrated in those areas of greatest organizational importance.

- The grouping of organizational issues into a number of broad categories, or headings, helped interpret the results, and appears to be a useful tool, even though the boundaries are not completely clear cut. In particular, the recognition that practical 'transitional issues', such as the timing of the implementation, the level of organizational disruption, and the decommissioning of the old system, have an organizational dimension is of interest.

- In some cases the task of classifying an issue as either organizational and technical was difficult. This problem became particularly apparent with regard to the issue of identifying systems interfaces. It is likely that this is a technical issue which has organizational implications, rather than being an organizational issue in its own right.

- Further research is needed to examine whether the groupings of organizational issues, proposed in this paper, can be developed into a more clear cut and robust framework. This will necessitate further clarification and verification of the distinction between organizational and technical issues. It can be argued that a strong theoretical framework of this kind is an essential pre-requisite before more practical work with regard to the treatment of organizational issues can be successfully tackled.

This research confirms the increasing importance of organizational issues, and the need to explicitly address them in the systems development process. There is, however, much further research, in this area, which still needs to be undertaken, especially with regard to the specification and treatment of organizational issues. This should ultimately lead to the development of a new generation of tools, methods and strategies for the effective treatment of organizational issues, and to the introduction of more appropriate ways training of IT professionals in organizational aspects of design.

In the short term, there is a need for further detailed study of what systems developers do and think. For example, this study found that some issues are given more attention than others, but it was not totally clear why this related to their perceived importance. Furthermore, work is needed to discover how these perceptions are formed and influenced, and how the link to the frequency of consideration operates. Such work may well need to examine what motivates systems developers to address certain issues in specific ways. It is also important to clarify precisely what systems developers do when they treat organizational issues 'implicitly', and the effectiveness of such attempts need evaluating. Of course, the effectiveness of different types of 'explicit' approaches to the treatment of these issues also need evaluating. This will require in-depth 
studies, interviewing a cross section of systems developers, and possibly longitudinal studies tracing a series of systems development projects from conception through to operation.

\section{Acknowledgements}

The authors would like to take the opportunity to thank all those IT professionals who took the time and trouble to respond to the questionnaire, especially those who were involved in the detailed pre-testing exercise. The authors would also like to thank Professor David Buchanan, and the two referees, for their many helpful comments and suggestions. 


\section{References}

Bloomfield, B. P. \& Coombs, R. 1992, Information Technology, Control and Power: The Centralisation and De-centrailisation Debate Revisited. Journal of Management Studies, 29, 459-484.

Buchanan, D. 1991, Figure-Ground Reversal in Systems Development \& Implementation: From $\mathrm{HCl}$ to OSI. in Nurminen, M. \& Weir, G. (eds) Human Jobs and Computer Interfaces, North Holland, 213226.

Clegg, C., Warr, P., Green, T., Monk. A.,Allison, G. \& Lansdale, M. 1989, People and Computers. How to Evaluate your Company's New Technology, Ellis Horwood, Chichester.

Clegg, C., Waterson, P. \& Clarey, N. 1994, Computer supported collaborative working: lessons from elsewhere, Journal of Information Technology, 9, 85-98.

Clegg, C. et al 1996, The performance of Information Technology and the role of human and organizational factors, Report to the ESRC.

Coombs, R. 1992, Organizational Politics and the Strategic Use of Information Technology, PICT Policy Research Paper No 20. ESRC.

Crowe, M. Beeby, R. \& Gammack, J. 1996, Constructing Systems and Information, McGraw-Hill, London.

Eason, K. 1988, Information Technology and Organizational Change, Taylor \& Francis, London.

Earl M. J. 1989, Management Strategies for Information Technology. Prentice Hall, Hemel Hempstead.

Ewusi-Mensah, K. \& Przasnyski, Z. 1991, On Information Systems Project Abandonment: An Exploratory Study of Organizational Practices. MIS Quarterly, March, 67-85.

Ewusi-Mensah, K. \& Przasnyski, Z. 1994, Factors Contributing to the Abandonment of Information Systems Development Projects. Journal of Information Technology, 9, 185-201.

Flaatten, P., McCubbrey, D., O'Riordan, P. \& Burgess, K. 1992, Foundations of Business Systems, The Dryden Press, Orlando.

Harrington, J. 1991, Organizational Structure \& Information Technology, Prentice Hall.

Hornby, C., Clegg, C., Robson, J., McClaren, C., Richardson, S. \& O’Brien, P. 1992, Human \& Organizational Issues in Information Systems Development. Behaviour \& Information Technology, 11, 160-174.

Kearney, A.T. 1990, Barriers to the Successful Application of Information Technology. Department of Trade and Industry and CIMA, London.

Lincoln, T. \& Shorrock, D. 1990, Cost-justifying current use of information technology, in Lincoln T. (ed). Managing Information Systems for Profit, John Wiley \& Sons.

Long, R. J. 1987, New Office Information Technology: Human and Managerial Implications, Croom Helm, London.

Lyytinen, K. and Hirscheim R. 1987, Information Systems Failures: A Survey and Classification of the Empirical Literature. Oxford Surveys in Information Technology. 4, 257-309.

Lucas H. C. 1975, Why Information Systems Fail. Columbia University Press, New York.

Markus M. and Robey D. 1983, The Organizational Validity of Management Information Systems. Human Relations. 36, 203-226.

Mumford, E. 1986, Using computers for business success: The ETHICS method, Manchester Business School, Manchester. 
Pliskin N., Romm T., Lee, A.\& Weber Y. 1993, Presumed versus Actual Organizational Culture: Managerial Implications for Implementation of Information Systems. The Computer Journal. 36, 143-152.

Preece, J. 1994, Human-Computer Interaction, Addison-Wesley, Wokingham.

Price Waterhouse, 1996, Information Technology Review 1995/1996, Price Waterhouse, London.

Raymond, L., Pare, G., and Bergeron, F. 1995, Matching information technology and organizational structure: an empirical study with implications for performance. European Journal of Information System, 4, 3-16.

Romm, T., Pliskin, N., Weber, Y. \& Lee, A. 1991, Identifying organizational culture clash in MIS implementation. Information \& Management, 21, 99-109.

Sauer C. 1993, Why Information Systems Fail: A Case Study Approach. Alfred Waller, Henley.

Schultz, R. \& Slevin, D. 1975, A program of research on implementation. In Implementing Operations Research / Management Science. in Schultz, R. \& Slevin, D. (eds), American Elsevier.

Stowell, F. \& West, D. 1994, Client-led Design, McGraw-Hill.

Thach, E. \& Woodman, R. 1994, Organizational Change and Information Technology: Managing on the Edge of Cyberspace. Organizational Dynamics, 23, 30-45.

Walton R. E. 1989, Up \& Running: Integrating I.T. and the Organization. Harvard Business School, Boston.

Willocks, L. 1990, Editorial: Theme issue introduction. Journal of Information Technology, 5, 182-184.

Willcocks. L \& Griffiths, 1994, Management and Risk Issues in Larger-Scale IT Projects: A Comparative Analysis. Oxford Institute of Information Management: Research \& Discussion Papers, RDP94/7.

Wong E. and Tate G. 1994, A Study of User Participation in Information Systems Development. Journal of Information Technology. 9, 51-60. 


\begin{tabular}{|c|c|c|c|}
\hline Perspective & Historical & Current & Future \\
\hline Primary & Increased & Plus Improved & Plus Strategic \\
\hline Benefits & Productivity & Decision-Making & Benefits \\
\hline \multirow[t]{3}{*}{ Focus } & Automate & Seek & Completely \\
\hline & Existing & Process & Reengineer \\
\hline & Processes & \multicolumn{2}{|c|}{ Improvements Organization } \\
\hline Scope & $\begin{array}{l}\text { Stand-alone } \\
\text { functional } \\
\text { applications }\end{array}$ & $\begin{array}{l}\text { Increased } \\
\text { integration }\end{array}$ & $\begin{array}{l}\text { High levels of } \\
\text { Integration }\end{array}$ \\
\hline \multirow[t]{2}{*}{ Environment } & Relatively & Increasingly & Highly \\
\hline & Stable & Dynamic & Dynamic \\
\hline $\begin{array}{l}\text { Systems } \\
\text { Development }\end{array}$ & $\begin{array}{l}\text { Highly } \\
\text { centralised }\end{array}$ & $\begin{array}{l}\text { Relatively } \\
\text { centralised }\end{array}$ & $\begin{array}{l}\text { Increasingly } \\
\text { decentralised }\end{array}$ \\
\hline $\begin{array}{l}\text { Information } \\
\text { Provision }\end{array}$ & $\begin{array}{l}\text { Highly } \\
\text { formalised }\end{array}$ & $\begin{array}{l}\text { Formal reports } \\
+ \text { ad hoc enquiries }\end{array}$ & $\begin{array}{l}\text { Highly } \\
\text { disseminated }\end{array}$ \\
\hline $\begin{array}{l}\text { Organizational } \\
\text { Impact }\end{array}$ & $\begin{array}{l}\text { Moderate } \\
\text { impact }\end{array}$ & $\begin{array}{l}\text { Strong } \\
\text { impact }\end{array}$ & $\begin{array}{l}\text { Very strong } \\
\text { impact }\end{array}$ \\
\hline
\end{tabular}




\begin{tabular}{llllll}
\hline Organizational Issue & Ave & Min & Max & 1 \& & $\mathbf{4}$ \& 5 \\
\hline Consider System's Interfaces & 4.3 & 2 & 5 & 4 & 54 \\
Undertake Cost-benefit Analysis & 4.1 & 2 & 5 & 4 & 54 \\
Align with Information systems strategy & 4.1 & 2 & 5 & 4 & 50 \\
Assess Training and education requirements & 4.0 & 2 & 5 & 5 & 46 \\
Prioritize work to focus on critical areas & 3.8 & 2 & 5 & 6 & 46 \\
Consider future needs of the organization & 3.6 & 2 & 5 & 8 & 36 \\
Review timing of implementation & 3.6 & 2 & 5 & 12 & 34 \\
Consider level of organizational disruption & 3.4 & 2 & 5 & 15 & 29 \\
Re-design jobs to suit system & 3.2 & 1 & 5 & 19 & 24 \\
Assess impact on organizational structure & 3.0 & 1 & 5 & 24 & 21 \\
Assess the system's impact on user motivation & 2.9 & 1 & 5 & 25 & 17 \\
Consider health and safety / ergonomic issues & 2.7 & 1 & 5 & 34 & 15 \\
Assess impact on organizational culture & 2.6 & 1 & 5 & 33 & 16 \\
Evaluate the impact on the distribution of power & 2.0 & 1 & 5 & 49 & 9 \\
\hline
\end{tabular}

Table2: The frequency with which organizational issues are addressed 


\begin{tabular}{lllllc}
\hline Organizational Issue & Ave & Min & Max & 1.5 & 11-14 \\
\hline Undertake Cost-benefit Analysis & 3.5 & 1 & 14 & 49 & 5 \\
Prioritize work to focus on critical areas & 5.1 & 1 & 13 & 33 & 4 \\
Consider future needs of the organization & 5.2 & 1 & 14 & 33 & 2 \\
Assess Training and education requirements & 5.4 & 1 & 11 & 30 & 2 \\
Align with Information systems strategy & 5.7 & 1 & 14 & 31 & 7 \\
Consider System's Interfaces & 6.0 & 1 & 13 & 28 & 8 \\
Re-design jobs to suit system & 6.4 & 1 & 14 & 23 & 4 \\
Review timing of implementation & 6.4 & 1 & 12 & 21 & 8 \\
Assess the system's impact on user motivation & 6.8 & 1 & 13 & 19 & 11 \\
Consider level of organizational disruption & 7.6 & 1 & 14 & 13 & 14 \\
Assess impact on organizational structure & 8.5 & 1 & 13 & 13 & 19 \\
Assess impact on organizational culture & 9.4 & 1 & 14 & 12 & 30 \\
Consider health and safety / ergonomic issues & 10.4 & 2 & 14 & 5 & 30 \\
Evaluate the impact on the distribution of power & 11.7 & 3 & 14 & 7 & 40 \\
\hline
\end{tabular}

Table3: The relative importance of organizational issues 


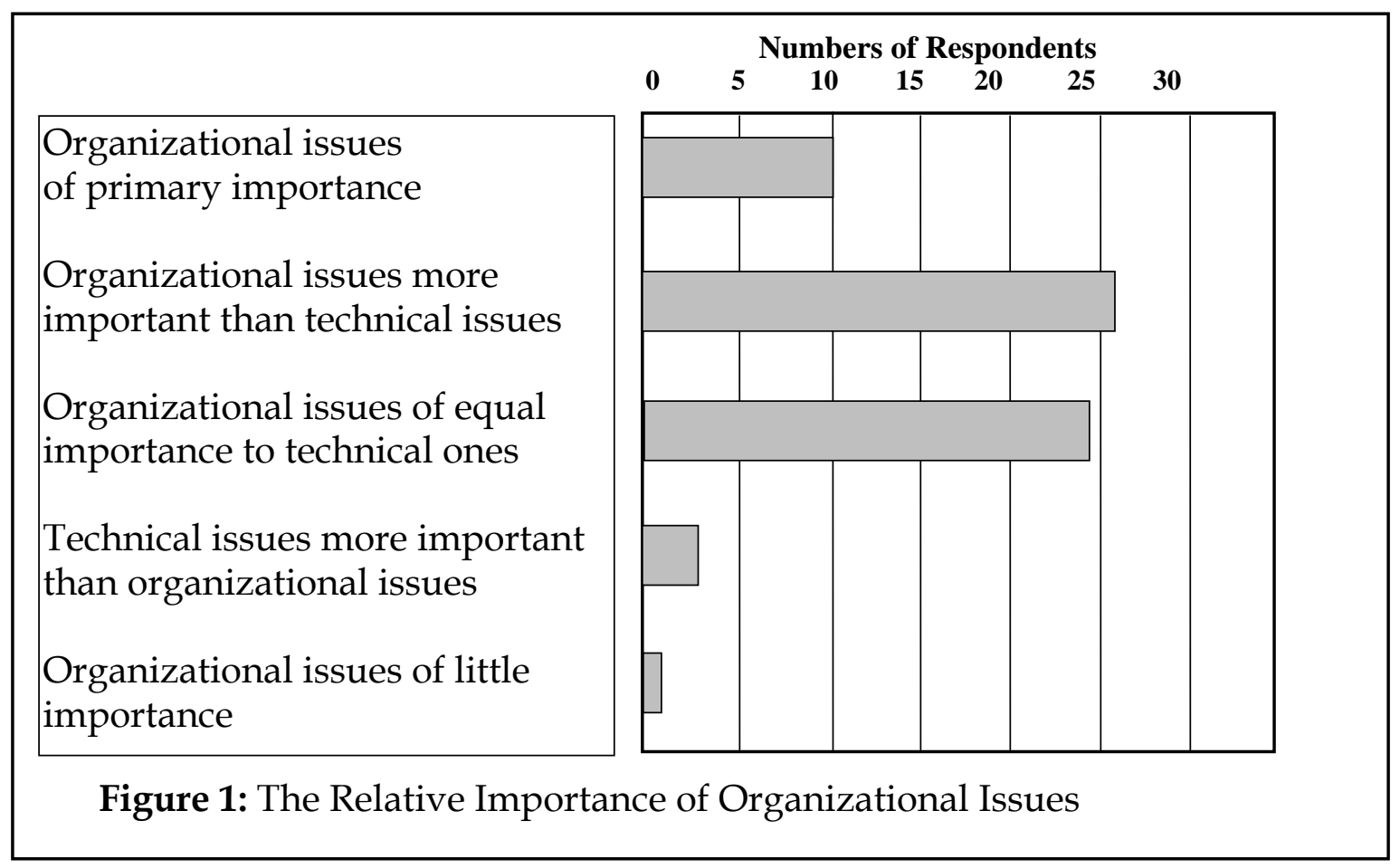




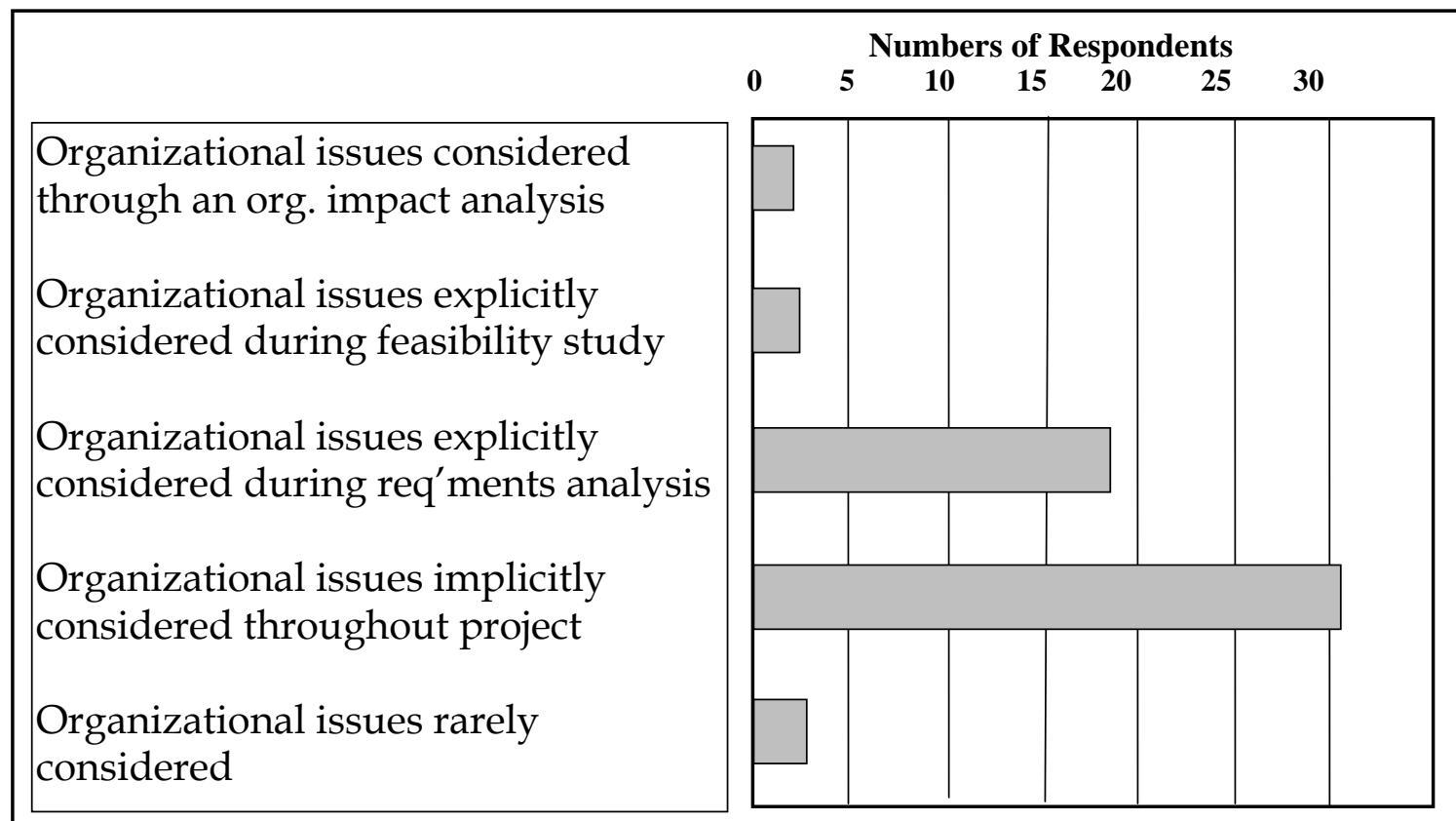

Figure 2: The Method of Treatment for Organizational Issues 


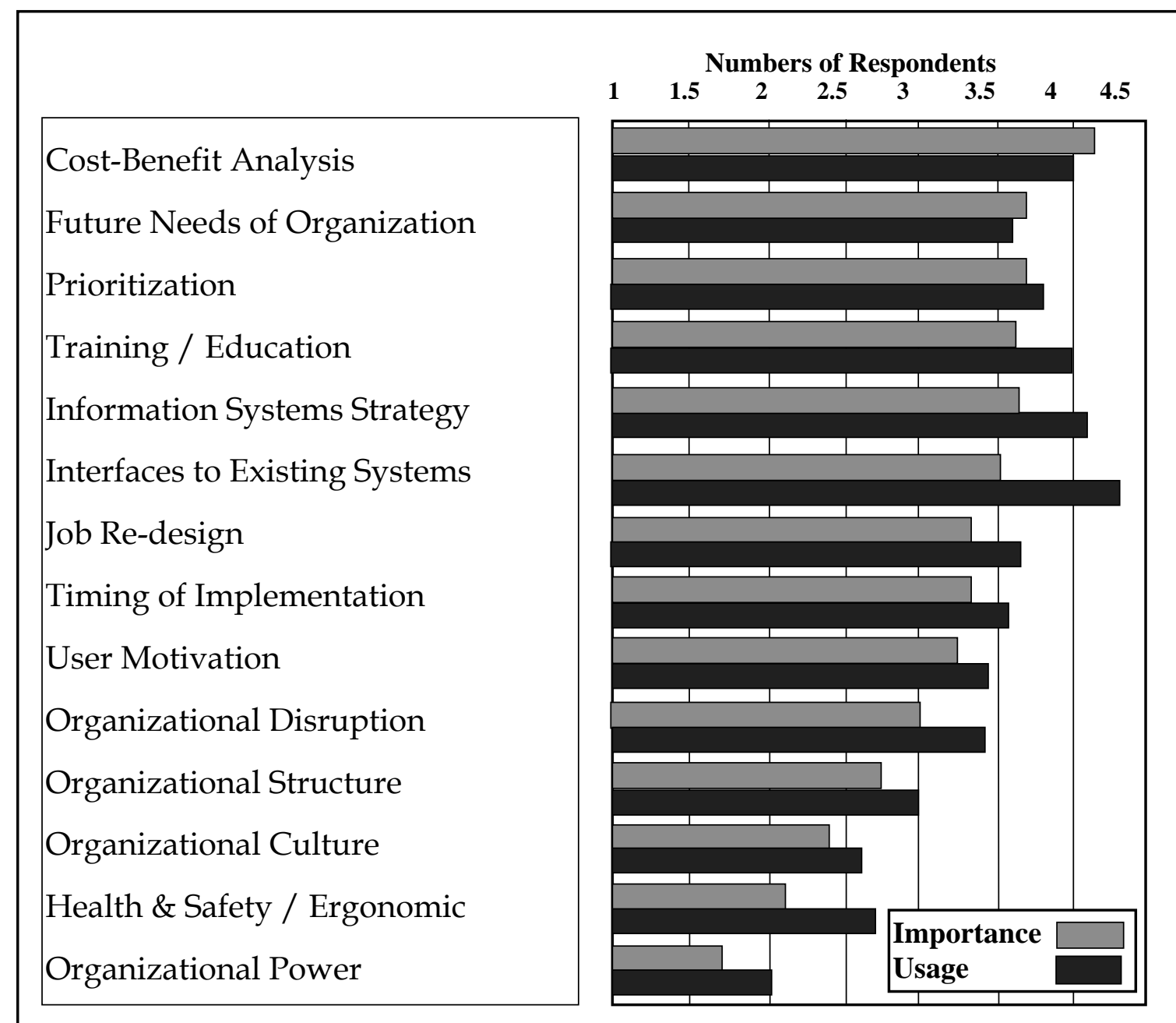

Figure 3: The Importance and Frequency of Treatment of Organizational Issues 